\title{
Construct-Inference-Process (CIP) Framework for UX Model Building and Evaluation Over Time
}

\author{
Azham Hussain ${ }^{1}$, Emmanuel O.C. Mkpojiogu², Fazillah Mohmad Kamal ${ }^{3 *}$ \\ ${ }^{1,2}$ School of Computing, Universiti Utara Malaysia, 06010 UUM, Sintok, Malaysia \\ ${ }^{2}$ Department of Computer and Information Technology, Veritas University, Abuja, Nigeria \\ ${ }^{3}$ School of Quantitative Sciences, Universiti Utara Malaysia, 06010 UUM, Sintok, Malaysia \\ Corresponding author: ${ }^{3 *}$ fazillah@uum.edu.my
}

Article History: Received: 10 November 2020; Revised: 12 January 2021; Accepted: 27 January 2021; Published online: 05 April 2021

\begin{abstract}
This paper used a literature review strategy to investigate the approaches used in building user experience (UX) models and in evaluating UX over time. The paper reports that there is no uniform and consensus approach in the strategies used in developing user experience models and in evaluating UX over time. Researchers adopt divergent ways in building UX models and evaluating UX. This makes the development of a common approach difficult. It is also shown that there are three frequently employed approaches in the development of UX models and in the evaluation of UX over time. The three approaches are the construct, inference and process (CIP) approaches. The first two are associated with UX model development while the third is associated with UX evaluation over time utilizing the developed model. Researchers seldom use the three approaches combined together due to the rare use of longitudinal research in UX domain. Consequently, this paper strongly recommends and proposes the use of the combination of the three approaches to build UX models and to employ the developed model in evaluating UX over time.
\end{abstract}

Keywords: Construct, evaluation, inference, model building, over time, process, user experience

\section{Introduction}

An interactive product simply put is generally any product that users sends input to and in turn it delivers a reasonable output as information to them (Carr, 2005). Equally, the ISO (ISO 9241-210 (2010) elaborated on interactive systems as the "combination of hardware, software and/or services that receives input from, and communicates output to users". Benyon, in addition, utilized the term 'interactive systems' to describe the products, and software systems, that handle the transmission, display, storage or transformation of information which users can perceive and respond or react to dynamically (Benyon, 2010). These are thus, systems that enable interaction between users and the systems in use. These interactive artifacts can be looked at from both the user and system perspectives (Carr, 2005). To the user, the artifact is an instrument for achieving tasks, it is a thing that can receive input, and display output in some ways (Samardžija, 2016; Hussain et al., 2018; 2019a; 2019b; Mkpojiogu et al., 2018; 2019). Interactive systems involve an important degree of user interaction. Physically, users interact with systems via some media of interaction like video input, keyboard, touch screen, voice recognition, mouse, and motion sensors, among others for haptic, verbal, and/or visual interaction etc. (Samardžija, 2016).

Hart (2014) explains that little studies have dwelt on the summative evaluations of interactive systems, and that even lesser attention is given to the interactive characteristics in such products. Comparable to user experience, interactivity is commonly used; however, the notion is rather unclear (Lee, 2005; Wu, 2006). The gain from interactivity and its impact on UX still remains unclear (Hart, 2014). Hart (2014) reiterates that research in human-computer interaction $(\mathrm{HCI})$ has principally concentrated on the use of interactivity to improve user experience in gaming and amusement domains, with a heavy stress on absorption and immersion (Korhonen et al., 2009; Sanders \& Cairns, 2010), presence and flow (Qiu \& Benbasat, 2005), and playfulness (Korhonen et al., 2009). Hart (2014) further stated that there is an increasing research focus in the use of interactive artifacts within cultural contexts to promote engageability (Haywood \& Cairns, 2006; Othman et al., 2011), and with a rise of interest in serious gamming, that harnesses the principles of fun, ludic and playful designs with interactive technological artifacts (Deterding et al., 2011). A few research works on the influence of interactivity in the electronic commerce sites domain have indicated that it can positively impact on user satisfaction, interest, motivation, pleasure, and enjoyment (Cyr et al., 2009; O’Brien, 2010). Nevertheless, these studies only investigated a limited set of quality perceptive attributes and so the effect of user experience attributes like those of aesthetics, emotion and usability in association to some particular interactive characteristics still remains unclear (Hart, 2014).

There is currently no agreement in the research domain on how UX should be designed or evaluated and whether the UX of a product can be accounted for by mere manipulation and measurement of the UX attributes 
(Law et al., 2014). This is further compounded by the dynamic features of UX. UX is temporal and there is a complex association among the several experiential moments or episodes of experience (Kashfi et al., 2017). UX is also dynamic. It develops and evolves in the course of time (Hassenzahl, 2010). For instance, over time, a user may perceive a novel feature in a product as old, or a complex feature as simple. Therefore, in the design and evaluation of UX, practitioners should give/pay good attention to the varying episodes of experience (Hassenzahl, 2010); such as, experience before use (expected experience), momentary experience (during usage), remembered experience (that is, shortly after usage) and accumulated experience (that is, over longer period of usage) (Hassenzahl, 2010). Practitioners need to decide which episodes are more significant than others for the software that is being designed, developed (or evaluated) and why; for example, for an electronic marketing site, first impression is more vital than it is for a work app. This understanding can then assist practitioners to suggest more appropriate design solutions. Also, first time users may be confused by the range of icons and options featured on the interface of some smart phones. Nevertheless, once they become familiar with the phones, they may become captivated and enthralled by the new features and possibilities that the devices present when compared to the usual features in phones. Hence, an experience is thus unique, very likely to evolve with time, and cannot be repeated (Hassenzahl, 2010; McCarthy \& Wright, 2004). After a first time experience, smartphone users who are novices will never experience again in their life time the first time encounter of smartphones usage again. The first time experience is now in the past (Kraus, 2017).

In addition, substantial UX studies have placed attention on short-term interactions and evaluations, hinged on users' first impressions, pre and post-interaction experiences; however, limited and scanty research works have captured user experience from long-term usage perspective (Hart, 2014). Even for the few works that captured long-term usage, the length of use varies (Hart, 2014). Prior studies have concentrated principally on short-term assessments, with users' first perceptions of digital artifacts (i.e., after a first time use), instead of in a long-term use. Studies show that the way a person perceives the quality of a product evolve with time. However, only a limited number of works showed such evolutions in usability, hedonics and aesthetics perceptions (Karapanos et al., 2008; von Wilamowitz-Moellendorff et al., 2006).

In UX research domain, there is no standard or common approach to building and evaluating UX over time. As such various researchers employ divergent strategies in doing this. It is vital to conceptualize a common approach to developing models for the evaluation of the user experience of interactive systems over time. This paper proposes a common and consensus strategy for the building and evaluation of user experience over time which hitherto is non-existent. There are three approaches to building models in UX for such purpose, they include: construct, inference, and process approaches. The first two approaches are concerned with model building while the third is for the evaluation of UX over time. Construct approach is an approach where latent/manifest constructs/qualities are used to build measurement, theoretical or evaluation models (Lavie \& Tractinsky, 2004; Tractinsky, 1997). This approach has to do with identifying and developing constructs/qualities for the assessment of UX (Porat \& Tractinsky, 2012). In UX several models have been developed by the construct approach. The inference approach on the other hand uses the relationships between constructs/qualities to build evaluation models (Hassesnzahl (2004). The approach is useful for examining the associations existing between constructs. This is to ensure that a relationship exit among the constructs/qualities. With this approach, the contribution or impact of a construct/quality on another can be assessed. The approach infers unavailable or unknown UX qualities from the available (or known) ones to infer into or better understand the overall quality of a product (Hassenzahl, 2004; Monk, 2004; Hassenzahl \& Monk, 2010).finally, the process approach is based on the cognitive process (it gains knowledge over time about the dynamic developments of the UX of a product). It is a panel or repeated measure or time series approach used to evaluate changes in perception and user judgment about a product over time from say, users' perceptions in the early stages of the process, when users form initial impressions about a product to later stages. This approach reveals the direction of relationships or effects in a model over time and can be used in evaluating UX dynamically over time. The process approach sees UX as a cognitive process that can be modeled and utilized to measure or assess the evolution in perception and judgment over time (Lindgaard et al., 2006, 2011; Tuch et al., 2012). It is used to observe, capture and evaluate UX at different time point over time (for example, before, during, and after interactions and in all time points). The approach is employed to assess the dynamic nature of UX over time (Lindgaard's et al., 2011; Porat \& Tractinsky, 2012). Other names for the process approach are panel, longitudinal, long-term, time series, or repeated measure approach. A combination of the three approaches builds and evaluates UX over time.In prior studies there is evidence that the three approaches are used in building UX models and for evaluating UX over time but not as a combined strategy. Constructs and inference approaches are often used together but with the process approach in combination. This is because most UX evaluation studies static evaluation studies (not dynamic).Static UX evaluation is a cross-sectional evaluation of UX at a one time period. 


\section{Methodology}

This present study used the survey of literature approach to discover and understand the various approaches used in building and evaluating user experience over time and sought to combine and harmonize them. Prior studies served as a seed to discovering this. The protocol used in the study is itemized thus: i) the downloading of relevant and appropriate literature sources on the approaches for building and evaluating UX over time; ii) synthesizingdownloaded sources for required information on the approaches for building and evaluating UX over time; iii) extracting the approaches for building and evaluating UX over time from relevant literature sources; iv) producing a harmonious and common framework for building and evaluating UX over time. Figure 1 indicates the research protocol for this study.

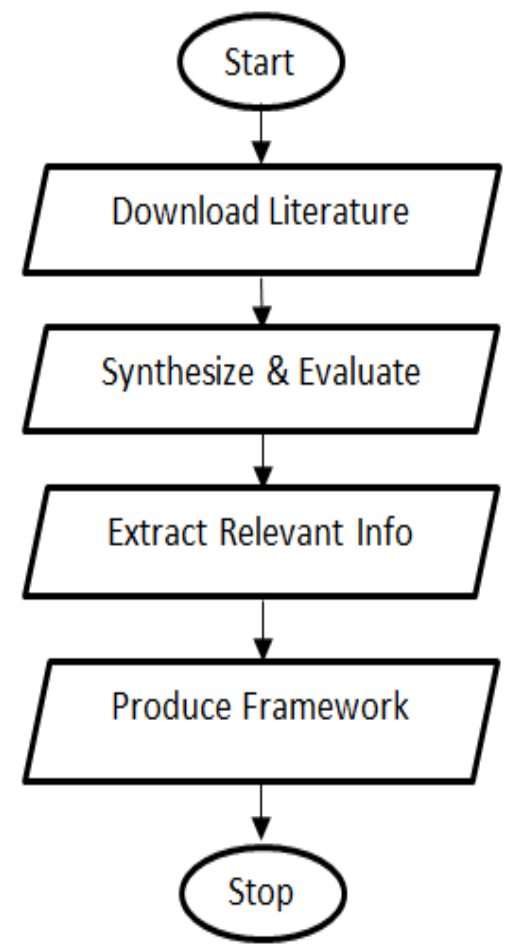

Figure 1. Research Protocol

\section{Results}

The findings of the literature survey on the approaches for building and evaluating UX over time reveal interesting outcomes. The findings show that construct, inference and process approaches are the most common, though the three are not often used in combination. Table 1 shows the frequency of occurrence.

Table 1. UX Model Building and Longitudinal Evaluation Approaches

\begin{tabular}{|l|l|l|l|}
\hline Authors & Construct & Inference & Process \\
\hline Lavie \& Tractinsky (2004) & $\sqrt{ }$ & & \\
\hline Tractinsky, 1997 & $\sqrt{ }$ & & \\
\hline Porat \& Tractinsky, 2012) & $\sqrt{ }$ & $\sqrt{ }$ & $\sqrt{ }$ \\
\hline Porat et al., 2007 & $\sqrt{ }$ & $\sqrt{ }$ & \\
\hline Hassenzehl (2004) & & $\sqrt{ }$ & $\sqrt{ }$ \\
\hline Hassenzahl \& Monk (2010) & & $\sqrt{ }$ & \\
\hline van Schaik et al. (2012) & & $\sqrt{ }$ & \\
\hline Diefenbach \& Hassenzahl (2011) & & $\sqrt{ }$ & \\
\hline Hartmann, Sutcliffe, \& De Angeli (2007) & & $\sqrt{ }$ & $\sqrt{ }$ \\
\hline Lindgaard, Fernandes, Dudek, \& Brown (2006) & & & $\sqrt{ }$ \\
\hline Lindgaard, Dudek, Sen, Sumegi, \& Noonan (2011) & $\sqrt{ }$ & $\sqrt{ }$ \\
\hline Tuch et al. (2012) & & & $\sqrt{ }$ \\
\hline Hartmann et al. (2008) & & & $\sqrt{ }$ \\
\hline Ou \& Sia (2010) & & & \\
\hline
\end{tabular}




\begin{tabular}{|l|l|l|l|}
\hline Mahlke \&Thüring (2007) & $\sqrt{ }$ & $\sqrt{ }$ \\
\hline Zajonc (1980) & & & $\sqrt{ }$ \\
\hline Metzger (2007) & & & $\sqrt{ }$ \\
\hline Sutcliffe (2009) & & & $\sqrt{ }$ \\
\hline
\end{tabular}

This study recommends the construct-Inference-Process (CIP) framework for the building and evaluation of the UX of interactive system over time. In the first part of the framework, the UX constructs (UX dimensions, quality attributes \& metrics (subjective measures)) are identified and synthesized. In the second part, the associations of /among the UX constructs (dimensions, quality attributes (criteria) \& the overall UX construct) are identified and synthesized. In the third part, to evaluate UX over time, the process approach is employed (Figure 2). This approach deals with the assessment of UX longitudinally over a several time points or continuous period of time to evaluate observed trends in UX (this also implies that data will be collected repeatedly and longitudinally within the time frame of the research). Since UX is dynamic, temporal, emerging and evolving over time, it will be insufficient to evaluate UX at one time point (i.e., a momentary or static UX). To obtain better and richer insight into the temporality and uniqueness of the UX of users and to unravel the different episodic landscapes (evolving stages and phases) in UX, an evaluation of UX over time is necessary.

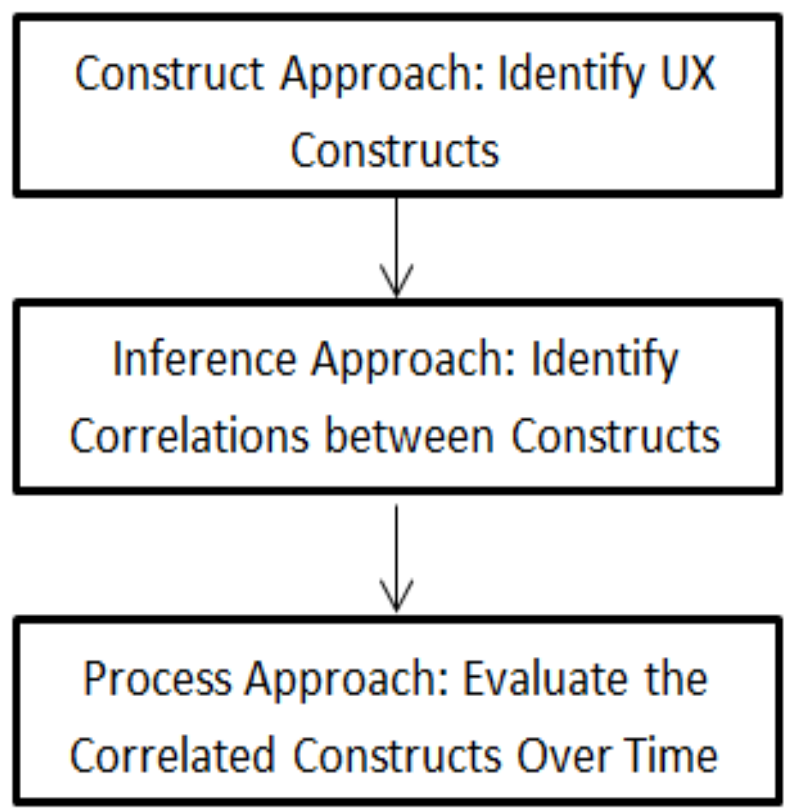

Figure 2. Framework for UX Model Building andthe Evaluation of UX over time

\section{Conclusion}

This study researched on the approaches for building and evaluating user experience over time. The paper observed that there is no standard and common strategy in building models and evaluating UX over time. Researchers adopt varying approaches to building UX models and evaluating UX. This makes uniformity and standardization difficult. It is also revealed that there are three commonly used approached in the building of UX models and in the evaluation of UX over time. These three approaches are the Construct, inference and process approaches. The first two relate to model building while the last and the third relates to UX evaluation over time using the built model. Researchers hardly employ a combination of the three approaches together since longitudinal studies of UX are rare. However, this study strongly recommends and proposes the use of the three approaches in combination to build UX models and to utilize the built model in assessing UX over time.

\section{References}

1. Benyon, D. (2010). Designing interactive systems: a comprehensive guide to HCI and interaction design. 2 ed. Harlow, England ; N.Y: Pearson Education Canada.

2. Carr, D.A. (2005). Introduction to interactive systems. http://www.sm.luth.se/csee/courses/smd/158/slides/Introduction2IS.pdf.

3. Cyr, D., Head, M., \& Ivanov, A. (2009). Perceived interactivity leading to e-loyalty: Development of a model for cognitive-affective user responses. International Journal of Human-Computer Studies, 67(10), 850-869. 
4. Deterding, S., Dixon, D., Khaled, R., \& Nacke, L. (2011). From Game Design Elements to Gamefulness: Defining "Gamification." In Proceedings of the 15th International AcademicMindTrek Conference: Envisioning Future Media Environments (pp. 9-15). New York, NY, USA: ACM.

5. Diefenbach, S., \& Hassenzahl, M. (2011). The dilemma of the hedonic - Appreciated, but hard to justify. Interacting with Computers, 23(5), 461-472.

6. Hart, J. (2014). Investigating user experience and user engagement for design. Doctoral Dissertation, Manchester University.

7. Hartmann, J., Sutcliffe, A., \& de Angeli, A. (2007).Investigating attractiveness in web user interfaces. In Proceedings of the SIGCHI conference on Human factors in computingsystems (pp. 387-396). ACM.

8. Hartmann, J., Sutcliffe, A., \& de Angeli, A. (2008).Towards a theory of user judgment of aesthetics and user Hassenzahl, M. (2004).The interplay of beauty, goodness, and usability in interactive products.Human-Computer Interaction, 19(4), 319-349.interface quality.ACM Transactions on Computer-Human Interaction(TOCHI), 15(4), 1-30.

9. Hassenzahl, M. (2010). Experience design: technology for all the right reasons. Synthesis Lectures on Human- Centered Informatics, 3(1), 1-95.

10. Hassenzahl, M., \& Monk, A. (2010).The inference of perceived usability from beauty. HumanComputer Interaction, 25(3), 235-260.

11. Haywood, N., \& Cairns, P. (2006).Engagement with an interactive museum exhibit. In People and Computers XIX-The Bigger Picture (pp. 113-129). Springer London.

12. Hussain, A., Mkpojiogu, E.O.C. \& Hassan, F. (2018). Dimensions and sub-dimensions for the evaluation of m- learning apps for children: A review. International Journal of Engineering \& Technology (IJET), 7 (3.20), 291-295.

13. Hussain, A., Mkpojiogu, E.O.C. \& Kutar, M. (2019a). The impact of software features' perceived importance on the perceived performance of software products' quality elements. Journal of Computational and Theoretical Nanoscience. 16 (5-6), 2135-2140.

14. Hussain, A., Shamala, P., \& Mkpojiogu, E.O.C. (2019b). The effect of software features' perceived importance on the observed performance of software product qualities. Journal of Advanced Research in Dynamical and Control Systems (JARDCS), 11(08-SI), 1076-1082.

15. ISO 9241:210 (2010). Ergonomics of human-system interaction - Part 210: Human-centred design for interactive systems," Int. Stand. Organ., (pp. 1-32).

16. Karapanos, E., Hassenzahl, M., \& Martens, J-.B.(2008). User experience over time. In CHI '08 extended abstracts on Human factors in computing systems (pp. 3561-3566). Florence, Italy: ACM.

17. Kashfi, P., \& Feldt, R. 2017). Integrating user experience practices into software development processes: implications of the UX characteristics. PeerJ Computer Science.

18. Korhonen, H., Montola, M., \& Arrasvuori, J. (2009).Understanding playful user experience through digital games. In International Conference on Designing Pleasurable Products andInterfaces (pp. 274-285).

19. Kraus, L. (2017). User experience with mobile security and privacy mechanisms. Doctoral Dissertation, Technischen Universit at Berlin.

20. Lavie, T., \& Tractinsky, N. (2004).Assessing dimensions of perceived visual aesthetics of web sites. International Journal of Human-Computer Studies, 60(3), 269-298.

21. Lee, T. (2005).The impact of perceptions of interactivity on customer trust and transaction intentions in mobile commerce.Journal of Electronic Commerce Research, 6(3), 165-180.

22. Lindgaard, G., Dudek, C., Sen, D., Sumegi, L., \& Noonan, P. (2011). An exploration of relations between visual appeal, trustworthiness and perceived usability of homepages. ACM Transactions on Computer-Human Interaction, 18(1), 1-30.

23. Lindgaard, G., Fernandes, G., Dudek, C., \& Brown, J. (2006). Attention web designers: You have 50 milliseconds to make a good first impression! Behaviour \& Information Technology, 25(2), 115-126.

24. Mahlke, S. \& Thüring, M. (2007).Studying antecedents of emotional experiences in interactive contexts. Proceedings CHI 2007, 915-918.

25. Metzger, M. (2007). Making sense of credibility on the web: models for evaluating online information and recommendations for future research. Journal of American Society for Information Science and Technology, 58(13), 2078-2091.

26. Mkpojiogu, E.O.C., Hashim, N.L., Hussain, A., \& Tan, K.L. (2019).The impact of user demographics on the perceived satisfaction and comfort of use of m-banking apps.International Journal of Innovative Technology and Exploring Engineering, 8(8S), 460-466. 
27. Mkpojiogu, E.O.C., Hussain, A., \& Hassan, F. (2018). A systematic review of usability quality attributes for the evaluation of mobile learning applications for children. ICAST 2018, AIP Conf. Proc. 2016, https://doi.org/10.1063/1.5055494

28. Monk, A. (2004). The product as a fixed-effect fallacy.Human-Computer Interaction, 19(4), 371375.

29. O'Brien, H. L. (2010). The influence of hedonic and utilitarian motivations on user engagement: The case of online shopping experiences. Interacting with Computers, 22(5), 344-352.

30. Othman, M. K., Petrie, H., \& Power, C. (2011). Engaging visitors in museums with technology: scales for the measurement of visitor and multimedia guide experience. In Human-Computer Interaction-INTERACT 2011 (pp. 92-99).Springer Berlin Heidelberg.

31. Ou, C. X., \& Sia, C. L. (2010). Consumer trust and distrust: An issue of website design. International Journal of Human-Computer Studies, 68(12), 913-934.

32. Porat, T., Liss, R., \& Tractinsky, N. (2007). E--stores design: the influence of E--store design and product type on consumers' emotions and attitudes. In J. Jacko (Ed.), Human-Computer Interaction. HCI Applications and Services (Vol. 4553, pp. 712-721).Springer Berlin Heidelberg.

33. Porat, T., \& Tractinsky, N. (2012).Its a pleasure buying here: the effects of web--store design on consumers emotions and attitudes. Human-Computer Interaction, 27(3), 235-276.

34. Qiu, L., \& Benbasat, I. (2005).An investigation into the effects of Text-To-Speech voice and 3D avatars on the perception of presence and flow of live help in electronic commerce. ACM Transactions on Computer-Human Interaction, 12(4), 329-355.

35. Samardzija, A.C. (2016). Measuring the success of the interactive mobile information system at the individual level of use.Doctoral Thesis, University of Zagred.

36. Sanders, T., \& Cairns, P. (2010).Time perception, immersion and music in videogames. In Proceedings of the 24th BCS Interaction Specialist Group Conference(pp. 160-167). British Computer Society.

37. Sutcliffe, A. (2009). Designing for user engagement: Aesthetic and attractive user interfaces. Synthesis Lectures on Human-Centered Informatics, 2(1), 1-55.

38. Tractinsky, N. (1997). Aesthetics and apparent usability: empirically assessing cultural and methodological issues. In Proceedings of the ACM SIGCHI Conference on Human factors incomputing systems (pp. 115-122). ACM.

39. Tuch, A. N., Roth, S. P., Hornbæk, K., Opwis, K., \& Bargas--Avila, J. A. (2012). Is beautiful really usable? Toward understanding the relation between usability, aesthetics, and affect in HCI.Computers in Human Behavior, 28(5), 1596-1607.

40. Van Schaik, P., Hassenzahl, M., \& Ling, J. (2012).User-experience from an inference perspective. In ACM Transactions on Computer-Human Interaction (TOCHI) (Vol. 19, p. 11).

41. Von Wilamowitz-Moellendorff, M., Hassenzahl, M., \& Platz, A. (2006). Dynamics of user experience : How the perceived quality of mobile phones changes over time. In User ExperienceTowards a unified view, Workshop at the 4th Nordic Conference on Human-Computer Interaction (pp. 74-78).

42. Wu, G. (2006). Conceptualizing and measuring the perceived interactivity of websites. Journal of Current Issues \& Research in Advertising, 28(1), 87-104.

43. Zajonc, R. (1980). Feeling and thinking: Preferences need no inferences. American Psychologist, $35(2), 151-175$. 